\title{
LE ELEZIONI IN ITALIA
}

a cura del CiSE (Aldo Paparo e Matteo Cataldi) 
Il 4 dicembre si è svolto il cruciale referendum confermativo sulla riforma costituzionale Renzi-Boschi cui il capo dell'esecutivo aveva indissolubilmente legato la propria esperienza di governo. Come noto, l'esito elettorale è stato davvero netto. Sostanzialmente un 60 a 40 per respingere la riforma costituzionale. Le conseguenze politiche immediate di questo sono ormai storia, con le dimissioni del governo Renzi e la nascita dell'esecutivo guidato da Gentiloni. In questo articolo analizziamo in profondità i risultati elettorali registrati il 4 dicembre, inserendoli nel contesto politico della legislatura in corso e tentando di spiegare come questi si siano venuti determinando.

\section{Il contesto}

Prima, è opportuno inquadrare il quesito referendario su cui gli elettori erano chiamati a pronunciarsi, e il contesto politico in cui la relativa campagna elettorale e poi il voto si sono svolti.

In quest'ottica è necessario descrivere brevemente i principali elementi della riforma costituzionale approvata dal Parlamento e poi respinta dagli elettori il 4 dicembre e il suo percorso di approvazione. La XVII legislatura si era aperta, all'indomani della non-vittoria del centro-sinistra guidato da Bersani come una legislatura volta alle riforme, specie dopo la rielezione di Napolitano alla Presidenza della Repubblica ${ }^{1}$. Il primo esecutivo della legislatura, quello presieduto da Letta e che godeva dell'appoggio di tutto l'allora PDL (ovvero anche di Berlusconi), aveva avviato un percorso straordinario di riforma al di fuori di quello delineato dall'articolo 138 della Costituzione e in modifica dello stesso, sul quale aveva trovato l'appoggio, oltre che delle forze di governo, anche di parte delle opposizioni - segnatamente Lega, SEL, FDI, e perfino il M5S aveva a volte votato con la maggioranza.

Il passaggio di consegne a Palazzo Chigi fra Letta e Renzi segnò anche un cambio di rotta su questo profilo, con la scelta di presentare la riforma costituzionale attraverso un disegno di legge costituzionale di iniziativa governativa da approvarsi secondo l'ordinario meccanismo descritto dall' articolo 138 del testo costituzionale. Con questa svolta, il perimetro di sostegno alla riforma si riduceva all'osso, limitato alla risicata area che sostiene il nuovo esecutivo con l'integrazione di Forza Italia - che nel frattempo è passata all'opposizione e non fa più parte dalla maggioranza che sostiene il governo.

Cominciava così, nell'aprile del 2014, con la presentazione al Senato del disegno di legge costituzionale a firma Renzi e Boschi l'iter parlamentare di approvazione di quella che sarebbe stata, nella sua versione definitiva, la riforma su cui si sono pronunciati gli elettori il 4 dicembre. La gestazione parlamentare è stata di due anni esatti, con l'approvazione in seconda lettura del testo finale alla Camera nell'aprile 2016. Da sottolineare come, durante i due anni di gestazione parlamentare, la riforma abbia perso il sostegno di Forza Italia, e sia stata dunque approvata nella sua ultima lettura dalle sole forze

1 Nel suo secondo discorso di insediamento, Napolitano ha ripetutamente menzionato la necessità di «riforma delle istituzioni rappresentative, dei rapporti tra Parlamento e governo, tra Stato e Regioni». 
di governo on i voti dei parlamentari verdiniani di ALA. Immediatamente la richiesta di referendum confermativo era avviata sia da attori istituzionali sia attraverso la raccolta delle necessarie firme.

Questa la breve cronaca della riforma. Ci preme in particolar modo evidenziare come il suo legame con l'andamento della legislatura sia inestricabile sin dall'avvio stesso di quest'ultima, e non sia dunque legato al referendum, momento elettorale nel quale si è indubbiamente rivelato con maggiore chiarezza e rilevanza.

Vediamo ora quali fossero i contenuti della riforma costituzionale. Il decreto legislativo recava le «disposizioni per il superamento del bicameralismo paritario, la riduzione del numero dei parlamentari, il contenimento dei costi di funzionamento delle istituzioni, la soppressione del CNEL e la revisione del titolo V della parte II della Costituzione»². Prendendo in esame i diversi piani, senza volere essere in alcun modo esaustivi, ma solo per inquadrare compiutamente il voto referendario che tra breve analizzeremo, possiamo dire che la riforma prevedeva un sostanziale cambiamento del Senato. Questo non votava più la fiducia al governo, né le leggi nell'iter ordinario; i suoi membri venivano drasticamente ridotti e non erano più direttamente eletti, ma erano Consiglieri regionali e sindaci. Conseguentemente, si prevedeva un parallelo rafforzamento del ruolo della Camera, che rimaneva l'unica rappresentante della nazione, l'unica a dare la fiducia al governo ed era in grado di approvare da sola la maggior parte delle leggi. Il CNEL veniva abolito, così come sparivano dal testo costituzionale le province. Infine, veniva rivista la divisione dei poteri fra Stato e regioni, con l'abolizione delle materie a potestà concorrente ed il trasferimento di alcune competenze dal livello regionale a quello nazionale ${ }^{3}$. Come accennato, il quesito referendario era unico, e chiedeva di approvare il testo di riforma costituzionale di cui venivano elencati, nei modi esposti sopra, i principali elementi.

Prima di procedere all'analisi dei risultati elettorali, vi è ancora un piano che è importante affrontare. È quello relativo alla campagna elettorale, e al suo inquadramento nel contesto politico della XVII legislatura. In quest'ottica è fondamentale presentare due concetti della letteratura elettorale che ci saranno molto utili per comprendere il referendum del 4 dicembre. Il primo è quello di ciclo elettorale. La letteratura comparata mostra come la popolarità dei governi abbia un andamento ciclico all'interno dell'arco della legislatura (Campbell 1960, Miller e Mackie 1973; Tufte 1975; Shugart 1995; Stimson 1976). Si inizia con una fase iniziale di cosiddetta "luna di miele", in cui la popolarità si mantiene sui livelli registrati al momento dell'insediamento o addirittura aumenta. La popolarità tende poi gradualmente a scemare lungo il corso della legislatura, fino a raggiungere il picco di minimo attorno alla metà del ciclo o poco dopo. I governi riescono poi tipicamente a fare segnare delle risalite nella fase finale della legislatura, con l'approssimarsi delle nuove elezioni politiche.

Se guardiamo al caso italiano, questa regolarità empirica è stata più volte confer-

2 Dicitura questa particolarmente rilevante perché avrebbe poi costituito il tanto discusso corpo centrale del quesito che gli elettori hanno trovato stampato sulla scheda il 4 dicembre.

3 Per un approfondimento dei contenuti e degli aspetti giuridici della riforma costituzionale si vedano Crainz e Fusaro (2016) e Zagrebelsky e Pallante (2016). 
mata, anche se sottolineando le difficoltà dei governi italiani a fare registrare la crescita in popolarità nel finale dei cicli elettorali (Bellucci 2006). Se ci concentriamo in particolare sul ciclo di questa XVII legislatura repubblicana, possiamo osservare come anche in questo caso specifico, la teoria del ciclo elettorale sia confermata. Il governo Renzi si insedia nel febbraio 2014 con un grande afflato di popolarità nei confronti in particolare del suo giovane Primo Ministro che parla il linguaggio della gente e promette riforme molto attese e di buon senso. La luna di miele fra il nuovo esecutivo e gli italiani è pienamente in essere quando si vota per le elezioni europee nel giugno 2014, ed i risultati elettorali del PD lo indicano chiaramente (De Sio, Emanuele e Maggini 2014). Dopodiché, la popolarità dell'esecutivo cala di oltre venti punti nel giro di pochi mesi, senza più risalire (Diamanti 2016). Ed i risultati elettorali si allineano. Si comincia nell'autunno quando il PD vince le regionali in Emilia-Romagna e Calabria, ma in elezioni caratterizzate da una partecipazione straordinariamente bassa (Cataldi, Emanuele e Maggini 2015). Si prosegue poi nella primavera successiva, quando il centro-sinistra comincia a fare segnare le prime battute d'arresto (Paparo e Cataldi 2016). Per arrivare infine alla primavera 2016, a sei mesi dal referendum costituzionale, quando le rumorose vittorie del M5S a Torino e Roma rivelano la ormai conclamata crisi di popolarità del governo di centro-sinistra in carica (Emanuele, Maggini e Paparo 2016).

Si possono sostenere numerose tesi per spiegare questo andamento nella popolarità del governo Renzi: il perdurare della crisi economica, l'incapacità dell'esecutivo di portare a casa importanti cambiamenti, le troppo alte aspettative alimentate al momento dell'insediamento. Tutti questi fattori avranno probabilmente contribuito. In ogni caso, come abbiamo cercato di mostrare, la popolarità dei governi tende a calare dopo la luna di miele iniziale, senza che vi debbano essere particolari ragioni. Quel che interessa qui rimarcare è che il referendum costituzionale oggetto di questo contributo si è svolto nel dicembre del 2016, a due anni e mezzo dall'insediamento del governo e a più di un anno dallo scadere naturale della legislatura. Insomma nel momento più difficile per la popolarità di un governo all'interno del ciclo della legislatura ${ }^{4}$. Certo, se gli elettori avessero votato esclusivamente sulla riforma costituzionale e i suoi contenuti, la popolarità del governo non sarebbe rilevante per spiegarne le scelte di voto. Ma poiché, come vedremo, numerosi elementi indicano che il voto è stato "politico", ecco che questa diventa un elemento cruciale.

Il secondo concetto che dobbiamo introdurre e discutere brevemente è quello di elezioni di secondo ordine (Reif e Schmitt 1980). Sono queste tutte le consultazioni in cui in gioco non vi sia il governo del paese. Ovvero tutti i tipi di elezioni locali, o quelle

4

In questo senso si possono anche interpretare i tentennamenti dell'esecutivo circa la data di svolgimento del referendum costituzionale, inizialmente previsto a inizio ottobre e poi rinviato fino a slittare ai primi di dicembre. Il tentativo potrebbe essere stato proprio quello di allontanare la consultazione il più possibile dal momento di minimo della popolarità dell'esecutivo e collocarlo nella fase di sua risalita. In questo senso, anche la redazione di una legge di bilancio estremamente generosa, così come la chiusura di alcune importanti partite (quale, ad esempio, quella relativa al rinnovo del contratto dei pubblici dipendenti) proprio nell'immediatezza del momento referendario, appaiono come comportamenti di un esecutivo che, con l'avvicinarsi del concludersi della legislatura, cerca di recuperare consensi. 
europee. La letteratura mostra come, in tali elezioni, partiti ed elettori cerchino in realtà di determinare risultati desiderati nell'arena che conta di più, cioè proprio quella del governo nazionale che non è direttamente investita dal voto. Ecco che quindi, empiricamente, si riscontrano alcune occorrenza tipiche in questa categoria di elezioni. Rispetto alle politiche, si tende a votare meno, per via del fatto che la posta in gioco alle elezioni è più bassa, e i partiti di governo tendono a venire penalizzati a favore dei partiti di opposizione (Marsh 1998; Schmitt 2005; Schmitt e Teperoglou 2015).

Il referendum costituzionale 2016 si presentava come un esempio perfetto di elezione di secondo ordine. Gli elettori erano chiamati a decidere su un aspetto certamente rilevante della vita politica del paese, quale quello relativo alla riforma di alcune delle regole fondamentali del funzionamento delle nostre istituzioni. Tuttavia, non si votava direttamente per la scelta del governo. Non vi erano poi candidature locali o comunque specifiche di quella elezione in grado di orientare le scelte degli elettori: il quesito era lo stesso in tutto il territorio nazionale. Infine, occorre evidenziare un ultimo punto peculiare del voto del 4 dicembre. Rispetto ad altre elezioni di secondo ordine, la posta in gioco al referendum, seppur non coincidente con quella delle elezioni politiche, non se ne discostava molto. Certamente vi si avvicinava ben di più che non in elezioni europee o locali. Anzi, per gli elettori favorevoli al governo Renzi, in buona sostanza la posta in gioco al referendum coincideva con quella delle politiche: ovvero la possibilità di avere al governo, all'indomani dell'elezione e in conseguenza di questa, la propria opzione preferita. Per coloro che invece non gradivano il governo Renzi, non vi era la possibilità di mettere immediatamente a Palazzo Chigi il proprio governo preferito attraverso il voto referendario. Tuttavia, era chiaramente in palio la possibilità di vedere andare a casa un governo sgradito, e avvicinare la prospettiva di nuove elezioni politiche in cui conquistare il governo del paese. Questo elemento, come vedremo, si dimostrerà cruciale per comprendere i risultati elettorali, e la partecipazione in particolare.

La letteratura relativa alle elezioni di secondo ordine ci suggerisce dunque che, in una consultazione come il referendum costituzionale, gli elettori cercheranno di utilizzare quel voto che in quel momento hanno a disposizione per mandare messaggi al governo nazionale. A questo si sommano le chiare scelte degli attori politici, sia del fronte del NO che di quello del SI, di rendere il voto referendario una scelta pro o contro il governo Renzi. A cominciare dal Presidente del Consiglio stesso che, molti mesi prima del voto referendario, addirittura in primavera, al momento della campagna elettorale per le comunali, aveva dichiarato che avrebbe lasciato il governo se gli elettori avessero rigettato quel progetto di riforma su cui il suo governo aveva così fortemente investito. Alla luce delle sconfitte delle comunali, e del chiaro momento di difficoltà per la popolarità del governo che queste segnalavano, la campagna per il SI aveva poi cercato di cambiare rotta.

La campagna elettorale va vista così. Come un faticoso e tardivo tentativo da parte del fronte del SI di portare l'attenzione sui contenuti della riforma, che godevano dell'apprezzamento se non della maggioranza del corpo elettorale, certamente di una frazione superiore a quella che invece apprezzava il governo Renzi (D'Alimonte 2016; Pagnoncelli 2016). Dall'altra parte, il fronte del NO soffiava sulla insoddisfazione verso il governo, presentando il voto del 4 dicembre come l'occasione per mandare a casa Renzi, 
argomento certamente di maggiore richiamo che non critiche puntuali ai contenuti della riforma. Favorito anche da alcuni elementi di fondo del voto referendario che abbiamo descritto sopra, quest'ultimo sembra essere stato maggiormente efficace, come il risultato elettorale mostra. Certamente alcune critiche specifiche alla riforma hanno determinato il giudizio di alcuni elettori, come timori per possibili derive autoritarie, specie in funzione del celebre "combinato disposto" con la legge elettorale Italicum. Tuttavia, numerosi elementi indicano come la contrarietà a Renzi e al suo governo abbiamo motivato assai di più questo risultato, in quello che è stato un vero e proprio voto "politico". Nel prossimo paragrafo, in cui ci concentreremo sul risultato, potremo osservarli con chiarezza.

\section{Il voto}

La Tabella 1 riporta i risultati complessivi e quelli aggregati a livello regionale. Come possiamo osservare, il risultato complessivo ha visto la vittoria del NO di circa 18 punti percentuali. Il NO ha infatti raccolto il $59,1 \%$ dei voti validi, contro il $40,9 \%$ del SI. Nel solo territorio nazionale, escludendo quindi il voto degli italiani residenti all'estero, la vittoria per il respingimento della riforma costituzionale è stata ancora più larga. Ha sfiorato i 20 punti percentuali, con il NO che ha raggiunto il $60 \%$, contro il $40 \%$ del SI. In considerazione anche dell'alta partecipazione elettorale (quasi due aventi diritto su tre si sono recati alle urne - il 65,5\%), i voti in più per il fronte contrario alla riforma costituzionale sfiorano i 6 milioni. La Tabella 1 segnala anche come il SI abbia vinto in sole 3 regioni, in aggiunta alla circoscrizione degli italiani all'estero, che è quella in cui il margine di vantaggio per la conferma della riforma costituzionale è stato di gran lunga il più alto: quasi 30 punti, con il SI al $65 \%$ e il NO al 35\%. Le tre regioni del territorio nazionale in cui la maggioranza dei votanti si è espressa per confermare la riforma sono il Trentino-Alto Adige, la Toscana e l'Emilia-Romagna, nell'ordine dato dai margini registrati a favore del SI. Comunque assai più ridotti, come accennato, di quello rilevato fra gli italiani all'estero. I vantaggi per il SI sono infatti compresi fra gli 8 punti del TrentinoAlto Adige e il punto scarso fatto segnare in Emilia-Romagna.

Nelle rimanenti 17 regioni a prevalere è stato invece il NO. Nelle due regioni dell'ex Zona Rossa in cui a non ha vinto il SI si registrano i valori minimi per il margine di vittoria del NO: due punti in Umbria e 10 nelle Marche. Al nord il vantaggio per il NO è più ampio: è di poco superiore ai 10 punti in Valle d'Aosta, Lombardia e Piemonte, mentre supera i 20 punti in Veneto, Friuli-Venezia Giulia e Liguria. Venendo infine alle regioni meridionali, le vittorie per il NO sono ovunque più larghe della più larga registrata altrove - con la sola eccezione del Molise in cui il NO vince di poco meno di 22 punti, in linea con le regioni settentrionali maggiormente contrarie alla riforma. I margini di vittoria per il NO sono poco meno di 30 punti in Lazio e Abruzzo, sono compresi fra i 32 e i 37 punti percentuali in Campania, Puglia, Basilicata e Calabria, e infine superano i 40 punti nelle isole. In pratica aumentano linearmente via via che ci si allontana da Roma. Nel complesso, nell'aggregato centro-meridionale a sud dell'ex Zona Rossa, il NO ha prevalso con il $67,4 \%$ contro il $57,3 \%$ raccolto al Nord e il $48,8 \%$ fatto segnare nell'ex Zona Rossa.

Anche dall'analisi dell'affluenza ricaviamo importanti indicazioni circa il voto 
referendario e la sua natura. Nel complesso dell'Italia (senza la circoscrizione estero), ha votato ben il $68,5 \%$. Una percentuale molto alta, più simile a quella registrata alle politiche del $2013(75,2 \%)$ che a quella registrata alle europee del $2014(58,7 \%)$. E tutto questo senza che sulla scheda comparissero partiti o candidati. Già questo dato da solo ci mostra come questa tornata referendaria sia stata percepita come un appuntamento di natura "politica", che ha fortemente mobilitato gli elettori. Basti osservare la partecipazione elettorale che si è registrata nelle due uniche precedenti consultazioni omologhe della storia della Repubblica, quella del 2001 e quella del 2006. Nel referendum costituzionale del 2006 votò il 53,8\% degli elettori italiani (sempre escludendo l'estero), quasi 15 punti in meno rispetto al 2016. In quello di cinque anni prima la percentuale di votanti fu addirittura pari al $35 \%$, oltre 30 punti in meno di quello di quest'anno. Certo, si trattava in entrambi i casi di congiunture politicamente molto diverse da quella in cui si è tenuto quest'ultimo. Nell'ottobre del 2001 alla prova referendaria andava la riforma del Titolo V della Costituzione approvata dal centro-sinistra negli ultimi mesi della XIII legislatura, quella dal 1996 al 2001 in cui godeva della maggioranza in Parlamento. Al momento in cui si svolse quel referendum, e la relativa campagna elettorale, Berlusconi aveva vinto le elezioni politiche del maggio 2001 e sedeva a Palazzo Chigi sostenuto da un'ampia maggioranza parlamentare. La riforma in questione, seppure approvata dalla coalizione avversa, non costituiva un pericolo per il centro-destra. Anzi, sembrava incorporare alcune domande storiche di una parte almeno della coalizione moderata, segnatamente della Lega, visto il chiaro impianto di decentramento che essa attuava. In buona sostanza, non ci furono sforzi di mobilitazione dei partiti per quella che non era una consultazione in cui essi si giocassero alcunché, e così appena poco più di un elettore su tre si recò alle urne nell'unica consultazione che abbia approvato una riforma costituzionale. Una riforma tanto poco illuminata che oggi, appena 15 anni dopo, nessuno se la intesti e tutti concordino sulla necessità di una sua revisione.

Cinque anni più tardi, nel giugno del 2006, gli elettori erano chiamati a confermare o respingere la riforma voluta da Berlusconi sul finire della XIV legislatura. Quando si svolse quel referendum, il centro-sinistra aveva appena vinto le elezioni politiche dell'aprile 2006 e Prodi era tornato dopo 8 anni a Palazzo Chigi. Quel referendum costituzionale fu combattuto come la battaglia finale per sconfiggere Berlusconi, che era stato cacciato dalla guida del paese, ma poteva ancora fare gravi danni alla democrazia italiana se la sua riforma costituzionale fosse entrata in vigore. Il centro-sinistra produsse una notevole campagna di mobilitazione presso i suoi elettori nel pieno della luna di miele per il nuovo governo, e la sconfitta della riforma fu addirittura, seppur di poco, più larga che in questo 2016. Anche perché il centro-destra non fu capace di riportare alle urne sulla riforma costituzionale tutti i suoi elettori delle politiche di appena due mesi prima. Si trovava in un momento di ripensamento della propria strategia, e non è che una vittoria al referendum avrebbe potuto riportare Berlusconi a Palazzo Chigi. In ogni caso, il carattere "politico" di quella consultazione referendaria è confermato dall'alta partecipazione elettorale (certo 10 punti meno del 2016, ma 20 in più dell'analogo referendum costituzionale di cinque anni prima), e dalla distribuzione geografica di quel voto. Il SI vinse infatti solo nelle due roccaforti del berlusconismo: la Lombardia e il Veneto. In pratica si votò pro o 
contro Berlusconi. Chiaramente, non con lo stesso entusiasmo presso gli elettorati delle due opposte coalizioni, visto il particolare momento in cui si svolse quel referendum che abbiamo descritto sopra.

Ed arriviamo dunque al 2016. Questo referendum costituzionale è stato chiaramente diverso da entrambi gli esempi storici che abbiamo ripercorso. Stavolta il governo che ha propugnato la riforma costituzionale è ancora in carica. Negli altri due no: era già stato sconfitto da elezioni politiche e rimpiazzato da uno della parte avversa. Il più forte legame con l'arena politica nazionale, al di là dei contenuti specifici della riforma e del relativo quesito referendario, sta già in questo elemento che prescinde da qualsiasi considerazione strategica degli attori politici. Che comunque, come abbiamo sottolineato sopra, ci sono state, ed hanno a loro volta contribuito a rinsaldare il legame fra esito del referendum e prosecuzione dell'esperienza del Governo Renzi. Questo quadro di riferimento va tenuto in considerazione se vogliamo comprendere come mai in questo referendum costituzionale si sia registrata una partecipazione elettorale così alta: al di sotto, ma sostanzialmente in linea con una elezione politica ${ }^{5}$.

Come in elezioni politiche si è votato molto di più nel Centro-nord che al Sud. In particolare in Veneto (con il 76,7\%), in Lombardia (74,2\%) e nella ex Zona Rossa - specialmente in Emilia-Romagna e in Toscana, rispettivamente con il 75,9\% e il 74,5\%. In tutte le altre regioni centro-settentrionali la partecipazione si è comunque attestata fra il 70 e il 74\%. Nel Centro-sud e nelle isole si è invece votato di meno, con la Calabria fanalino di coda della partecipazione $(54,4 \%)$. Come già osservato in riferimento alle percentuali ottenute dal SI, anche in riferimento alla partecipazione notiamo come il valore massimo registrato nel Centro-sud sia inferiore a quello minimo nel resto d'Italia. E stavolta senza eccezioni. Il 69,7\% di affluenza in Liguria è infatti superiore al 69,2\% del Lazio. Come detto, questa minore affluenza al Sud è in linea con le aspettative, dal momento che storicamente il Sud partecipa di meno rispetto al resto del paese alle elezioni politiche (ed europee), così come è avvenuto anche al referendum del 2006. Ma non sempre in elezioni di carattere non politico-nazionale, come ad esempio le comunali o le regionali.

Ciò detto, la partecipazione al Sud è stata comunque ragguardevole (il 61,6\%), nettamente superiore a quella registrata nel referendum del 2006. Basti pensare che allora solo in Abruzzo e nel Lazio l'affluenza superò (di poco) il 50\%, mentre oggi il 54,4\%

La partecipazione elettorale degli elettori italiani è calata di oltre 5 punti fra le politiche del 2008 e quelle del 2013: dall' $80,5 \%$ al $75,2 \%$. Un analogo calo porterebbe l'affluenza delle prossime elezioni politiche poco al di sotto del $70 \%$. Un analogo tasso di variazione $(-6,6 \%)$ lo porterebbe al $70,2 \%$. Certo, il referendum costituzionale non si è svolto a cinque anni di distanza dalle politiche del 2013, ma a meno di quattro. Per cui, se elezioni politiche si fossero svolte il 4 dicembre, la stima della possibile partecipazione sarebbe probabilmente più vicina al $71 \%$ che non al 70. D'altronde però, fra europee 2009 ed europee 2014, la partecipazione è calata maggiormente: dal $66.5 \%$ al $58,7 \%$. Un calo di quasi 8 punti, pari al $-12 \%$. Sembra quindi che il calo della partecipazione si sia accentuato dopo il 2013, che potrebbe davvero portare l'affluenza delle prossime politiche molto vicina a quella del referendum. In ogni caso, una stima esatta della partecipazione elettorale alle prossime elezioni politiche, o in caso esse si fossero svolte il 4 dicembre, va oltre lo scopo di questo articolo. Qui interessa semplicemente rilevare che il $68,5 \%$ è probabilmente più vicino alla partecipazione elettorale che si sarebbe riscontrata in elezioni politiche vere e proprie di quanto il confronto con il $75,2 \%$ del 2013 non faccia sembrare. 
della Calabria è stato il dato dell'affluenza più basso.

TAB. 1 - Risultati elettorali del referendum costituzionale nelle diverse regioni.

\begin{tabular}{|c|c|c|c|c|c|c|}
\hline & Affluenza \% & Voti SI & Voti NO & $\% \mathrm{SI}$ & $\% \mathrm{NO}$ & Vantaggio NO \\
\hline Valle d'Aosta & 71,9 & 30,568 & 40,116 & 43,2 & 56,8 & 13,5 \\
\hline Piemonte & 72,0 & $1,054,749$ & $1,368,801$ & 43,5 & 56,5 & 12,9 \\
\hline Liguria & 69,7 & 342,671 & 515,777 & 39,9 & 60,1 & 20,2 \\
\hline Lombardia & 74,2 & $2,452,936$ & $3,058,210$ & 44,5 & 55,5 & 11,0 \\
\hline Veneto & 76,7 & $1,078,561$ & $1,756,466$ & 38,0 & 62,0 & 23,9 \\
\hline Friuli-Venezia Giulia & 72,5 & 267,357 & 417,754 & 39,0 & 61,0 & 22,0 \\
\hline Trentino-Alto Adige & 72,2 & 305,322 & 261,473 & 53,9 & 46,1 & $-7,7$ \\
\hline Emilia-Romagna & 75,9 & $1,262,484$ & $1,242,992$ & 50,4 & 49,6 & $-0,8$ \\
\hline Toscana & 74,5 & $1,105,769$ & $1,000,008$ & 52,5 & 47,5 & $-5,0$ \\
\hline Umbria & 73,5 & 240,346 & 251,908 & 48,8 & 51,2 & 2,3 \\
\hline Marche & 72,8 & 385,768 & 472,765 & 44,9 & 55,1 & 10,1 \\
\hline Lazio & 69,2 & $1,108,768$ & $1,914,397$ & 36,7 & 63,3 & 26,6 \\
\hline Abruzzo & 68,7 & 255,001 & 461,188 & 35,6 & 64,4 & 28,8 \\
\hline Molise & 63,9 & 63,695 & 98,728 & 39,2 & 60,8 & 21,6 \\
\hline Campania & 58,9 & 839,692 & $1,827,768$ & 31,5 & 68,5 & 37,0 \\
\hline Puglia & 61,7 & 659,354 & $1,348,573$ & 32,8 & 67,2 & 34,3 \\
\hline Basilicata & 62,9 & 98,924 & 191,081 & 34,1 & 65,9 & 31,8 \\
\hline Calabria & 54,4 & 276,214 & 561,726 & 33,0 & 67,0 & 34,1 \\
\hline Sicilia & 56,7 & 642,713 & $1,620,095$ & 28,4 & 71,6 & 43,2 \\
\hline Sardegna & 62,5 & 237,280 & 616,791 & 27,8 & 72,2 & 44,4 \\
\hline Totale Italia & 68,5 & $12,708,172$ & $19,026,617$ & 40,0 & 60,0 & 19,9 \\
\hline Estero & 30,7 & 722.915 & 394.408 & 64,7 & 35,3 & $-29,4$ \\
\hline Totale Italia ed Estero & 65,5 & 13.431 .087 & 19.421 .025 & 40,9 & 59,1 & 18,2 \\
\hline
\end{tabular}

La Figura 1 mostra la partecipazione elettorale a livello comunale. Ciascun comune è colorato tanto più scuro quanto più alta è stata l'affluenza al referendum costituzionale. Possiamo innanzitutto notare un generalizzato calo nell'affluenza procedendo da Nord a Sud chiaramente indicato dal progressivo schiarirsi delle tonalità.

Le zone più scure, quelle con più alta partecipazione, superiore a tre elettori su quattro, si concentrano nella provincia di Trento (10 punti in più della corregionale Bolzano), nella Lombardia centro-orientale (Lecco, Monza, Bergamo, e Brescia), in Veneto, 
in Emilia-Romagna (ad eccezione di Parma e Piacenza), e nel cuore rosso della Toscana (Firenze, Siena, Pisa, Arezzo). Allontanandosi da questo nucleo centro-settentrionale la partecipazione elettorale va via via scemando in maniera assai uniforme. Si mantiene abbastanza alta nel resto del Nord e nella cintura rappresentata da Marche, Umbria e alto Lazio: con valori medi compresi fra il 70 e il $75 \%$. L'affluenza è via via sempre più bassa mano a mano che si scende verso Sud lungo la penisola fino alla estremità della Calabria, come detto la regione con la partecipazione più bassa, anche se si segnalano dei comuni in controtendenza nelle province di Catanzaro e Cosenza. La Sicilia non è poi molto distante, ma si salva grazie alle affluenze attorno al $60 \%$ registrate a Ragusa e Catania. L'altra regione insulare, la Sardegna, fa invece segnare tassi di partecipazione in linea con la propria collocazione sulla direttrice Nord-Sud, analoghi a quelli di Campania e Puglia.

La mappa mostrata nella Figura 1 evidenzia anche, come una visibile macchia chiara, la porzione del paese a cavallo fra Marche, Umbria ed Abruzzo in cui la partecipazione elettorale rilevata è assai più bassa delle zone limitrofe per via delle inevitabili difficoltà logistiche nelle aree colpite dai recenti terremoti di agosto ed ottobre ${ }^{6}$.

6 Per la precisione, in quattro comuni delle Marche non si è votato. Questi sono Arquata del Tronto, Acquacanina, Castelsantangelo sul Nera e Ussita. In tutte le mappe mostrate in questo articolo i perimetri di tali comuni, per cui dunque non esistono i dati elettorali del referendum costituzionale, sono colorati di bianco. Comunque, come si può apprezzare dalla mappa in Figura 1, l'area bianca della bassa partecipazione si estende anche oltre la regione Marche, in Lazio e Umbria. Questo conferma come la macchia bianca della mappa che abbiamo indicato sia solo marginalmente dovuta ai quattro comuni non al voto, e che le conseguenze dei fenomeni tellurici si siano fatte sentire sulla partecipazione elettorale di una porzione ben più vasta del centro Italia. 
FIG. 1 - Mappa della partecipazione elettorale al referendum costituzionale per comune.

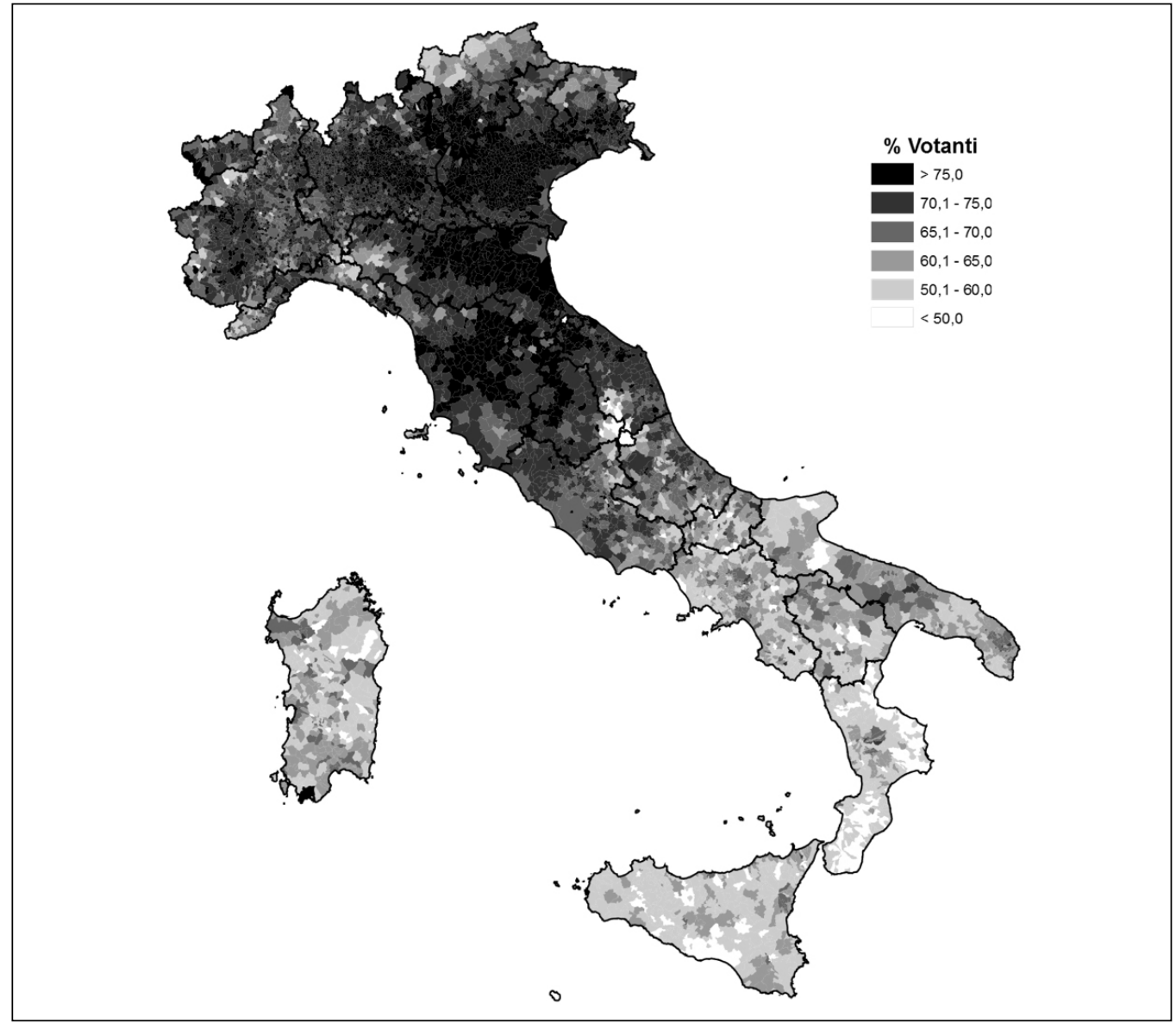

Un confronto interessante è quello con la partecipazione registrata alle elezioni europee del 2014. Come accennato sopra, rispetto alle europee l'affluenza è complessivamente aumentata di 10 punti. Ma ciò è avvenuto in maniera omogenea in tutto il paese? La risposta è contenuta nella Figura 2, che mostra le variazioni nella partecipazione fra 2014 e 2016 a livello di comune, ed è negativa. Infatti in alcuni comuni (quelli dal colore chiaro), l'affluenza è addirittura diminuita rispetto alle europee: come si vede dalla mappa, si trovano soprattutto in Piemonte, Lombardia, Toscana, Umbria, Marche e in alcune zone del Mezzogiorno.

Le tre tonalità di colore più scuro indicano, al contrario, i comuni dove l'affluenza è aumentata più della media nazionale. Come si vede, questi sono concentrati in Valle d'Aosta, Trentino-Alto Adige, Veneto, Friuli, Sardegna e in alcune aree della Sicilia, della Basilicata e della Puglia. Si tratta di un aumento della partecipazione al voto "spettacolare", che spesso supera i 20 punti percentuali e talvolta addirittura i 30 punti. Ciò significa che molti elettori che avevano giudicato irrilevante la contesa delle europee, hanno invece considerato molto rilevante questa tornata referendaria, confermando ancora una volta il carattere "politico" di questa consultazione. 
A tal proposito, infine, è da sottolineare come in alcune di queste zone netta sia stata l'affermazione del NO. La Sardegna ad esempio è la regione caratterizzata dalla più omogenea crescita dell'affluenza ed è anche la regione dove più alta è stata la percentuale del NO (72,2\%). Subito dopo viene la Sicilia (con il NO al 71,6\%) e in molte sue aree, come si è detto, l'affluenza è cresciuta notevolmente. Lo stesso avviene in altre aree del Mezzogiorno, dove la percentuale del NO è superiore alla media nazionale (così come in una certa misura in Veneto). Ciò non significa che gli elettori che si erano astenuti alle elezioni europee ed hanno invece votato al referendum abbiano votato tutti NO, tuttavia è un dato significativo di cui tenere conto ${ }^{7}$. Col senno di poi, è chiaro che un'affluenza molto alta ha sfavorito il fronte del SI, come del resto alcune analisi basate su sondaggi pre-elettorali avevano preconizzato. Infatti, l'alta affluenza è stata il frutto di una forte mobilitazione di tutti gli elettorati dei diversi partiti e i partiti che sostenevano le ragioni del NO erano dal punto di vista numerico nettamente superiori a quelli del fronte del SI.

$7 \quad$ Il comportamento degli astenuti 2013 può essere valutato solo sulla base dell'analisi dei dati individuali e dei flussi elettorali per non incorrere nella cosiddetta fallacia ecologica. È esattamente ciò che faremo, per alcune grandi e importanti città, nel prossimo paragrafo. 
FIG. 2 - Mappa della variazione nella partecipazione elettorale fra europee 2014 e referendum 2016.

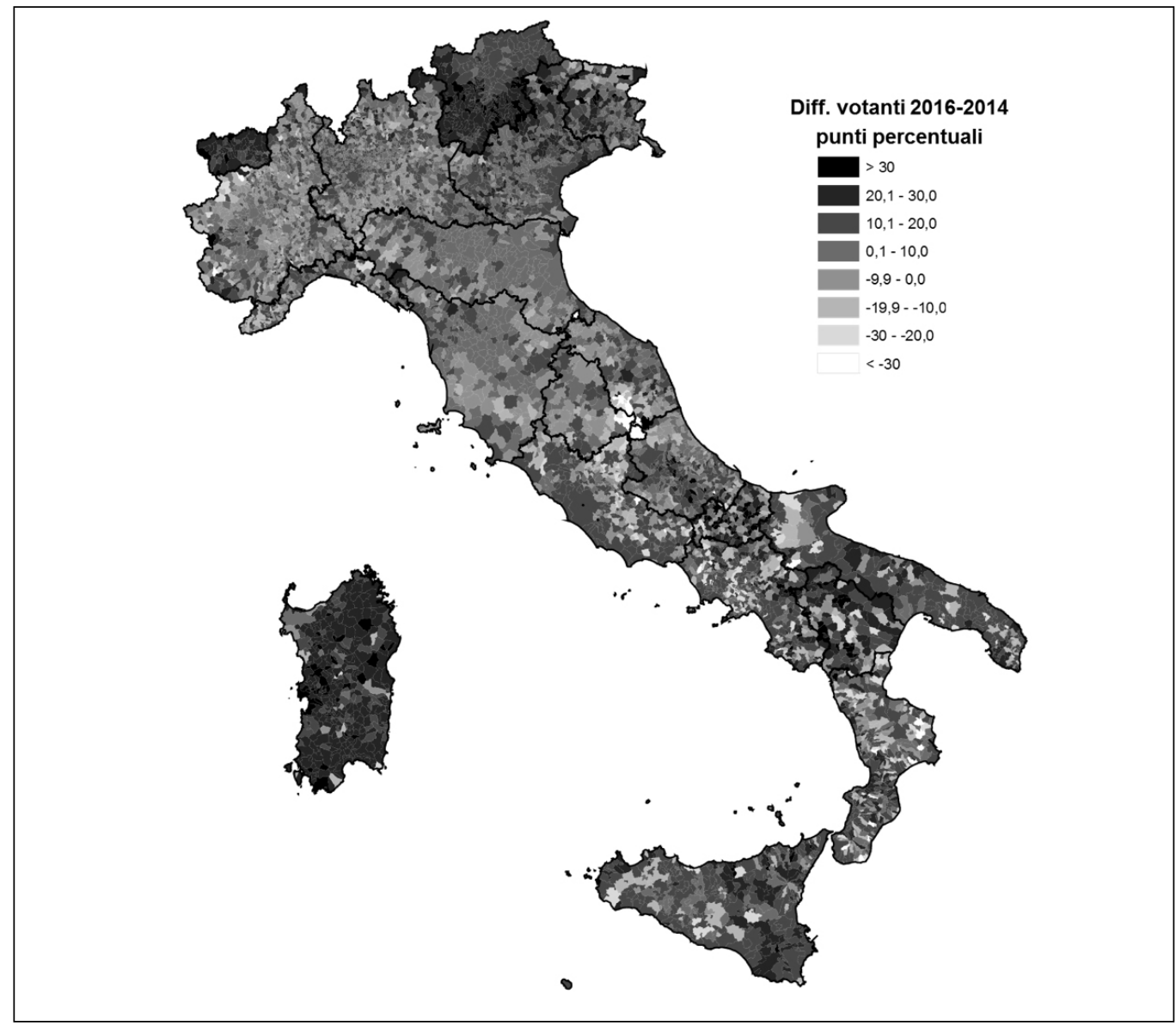

Abbiamo già avuto modo di descrivere i principali caratteri geografici del risultato elettorale del referendum costituzionale del 4 dicembre osservando il dettaglio per regione riportato nella Tabella 1. La Figura 3, tuttavia, ci consente di indagare molto più in profondità la geografia del risultato elettorale. Essa mostra, per ciascun comune, lo scarto registrato fra i risultati percentuali del SI e del NO. Anche in questo caso possiamo osservare innanzitutto la grande omogeneità geografica del risultato osservato. Si notano con chiarezza i toni scuri che indicano successi del SI nella provincia di Bolzano, in Toscana (soprattutto nelle province di Firenze e Siena, poi via via sempre meno, con il NO avanti nelle province più periferiche lungo la costa), ed in Emilia-Romagna (con la vistosa eccezione della provincia di Piacenza, dove il NO ha prevalso di 15 punti; ma anche nelle ulteriori tre province più periferiche - Rimini, Forlì e Parma - ha vinto il NO). Le uniche macchie scure di una certa rilevanza si riscontrano poi nella porzione dell'Umbria a confine della Toscana. Per il resto, i colori chiari delle vittorie del NO emergono dappertutto. Non troppo intenso nel resto della ex Zona Rossa. Mediamente un po' più forte in Lom- 
bardia e Piemonte, ma ancora contenuto. Già più in linea con il risultato medio nazionale in Veneto ed anche Liguria e Friuli-Venezia Giulia. La mappa mostra poi il progressivo schiarirsi dell'intensità del grigio, che indica una più larga vittoria del NO, nel Centrosud e in particolare nelle regioni insulari. Insomma, una mappa molto simile a quella del PD, integrato dalla SVP.

La Figura 3, inoltre, mostra alcuni ulteriori elementi di rilievo. Innanzitutto, pur mantenendo una notevole omogeneità geografica, si mostra più frastagliata dell'analoga mappa relativa all'affluenza. In altre parole, mentre la partecipazione è stata estremamente uniforme geograficamente, il voto lo è stato meno. In particolare si evidenziano sporadici puntini scuri, comuni in cui a prevalere è stato dunque il SI, un po' in tutte le regioni, e perfino in quelle in cui la vittoria del NO è stata più netta, come la Sicilia o la Sardegna. Per avere una conferma più sistematica di questa impressione emersa dalla osservazione delle mappe, abbiamo calcolato l'indice di autocorrelazione spaziale di Moran (Moran 1950) per la distribuzione geografica della partecipazione elettorale e dei risultati del SI a livello di comune. Questo indice sintetizza la omogeneità geografica della distribuzione delle variabili analizzate. Per l'affluenza il valore dell'indice di Moran è 0,74 ; per il voto al SI è pari a 0,56. Questo conferma che in effetti la partecipazione è stata distribuita più omogeneamente. Torniamo ora alla Figura 3 e cerchiamo di comprendere quali siano questi comuni che si differenziano da quelli circostanti. Si vede chiaramente Roma, in cui il NO ha vinto ma con un margine assai più ridotto che non nel resto del Lazio. Si nota poi Milano: insieme a Monza e qualche comune della cintura urbana rappresenta una macchia scura nella Lombardia chiara. Si apprezzano poi alcuni comuni del Salernitano dove, presumibilmente, il forte sostegno alla riforma del governatore De Luca può avere giocato un ruolo determinante. 


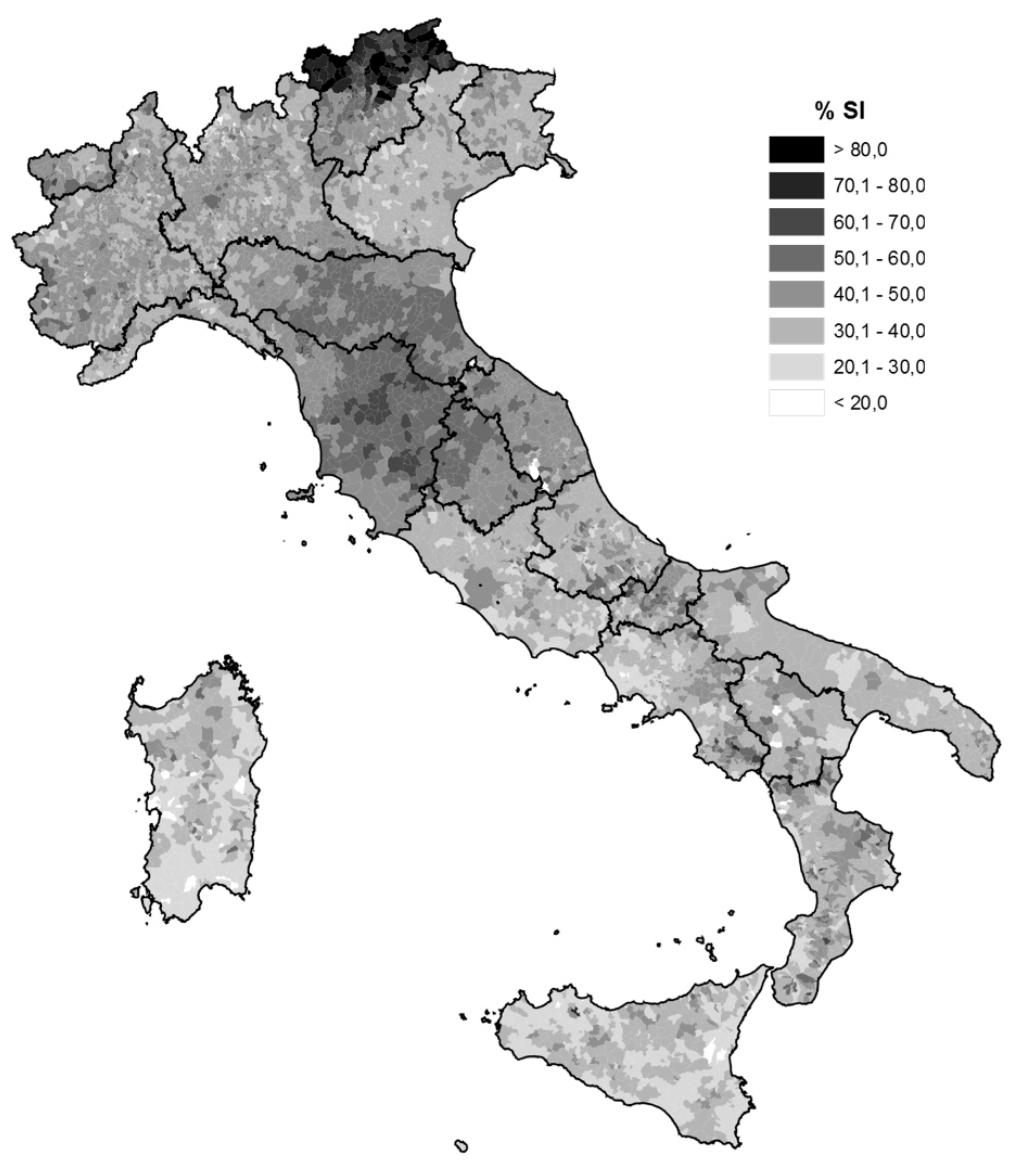

Insomma, la geografia del voto sembra proprio indicare come questa consultazione referendaria abbia avuto chiari caratteri di un voto "politico". Per interpretarlo al meglio, un confronto interessante che possiamo fare è quello con le europee del 2014. Infatti, il fronte del SI poteva contare su un totale di 12,6 milioni di voti raccolti alle europee dai partiti che la riforma l'avevano approvata (PD, NCD-UDC e SE). Una cifra straordinariamente simile ai 12,7 milioni di preferenze ottenute dal SI al referendum costituzionale ${ }^{8}$. Appare dunque rilevante indagare in che misura i voti del SI coincidano con quelli dei partiti di riferimento nel 2014. È proprio per comprendere ciò che mostriamo la Figura 4. In essa, i comuni colorati di scuro sono quelli il cui il SI è andato oltre la somma dei voti 2014 di PD, NCD-UDC e SE. I comuni invece colorati di chiaro sono quelli in cui alle europee i tre partiti hanno raccolto più voti di quelli del SI al referendum.

8 Naturalmente, per via della differente partecipazione nelle due elezioni in questione, in congiunzione anche con i diversi elettorati passivi, questi insiemi così simili valgono percentuali molto diverse: il $40 \%$ al referendum, quasi il $46 \%$ alle europee. 
Le sole macchie scure apprezzabili nella mappa sono quelle delle regioni a statuto speciale Trentino-Alto Adige e Valle d'Aosta. Occorre sottolineare come in queste due regioni la partecipazione sia aumentata di oltre 20 punti fra europee e referendum, come mostrato dalla Fig. 2: oltre il doppio della media nazionale. Questo ha certamente favorito le omogenee crescite del fronte del SI di oltre il 20\% rispetto ai risultati di PD, NCD-UDC e SE. Inoltre l'endorsement per il SI deciso dalla SVP, che non aveva invece votato la riforma e non fa per questo parte del fronte del SI alle europee da noi calcolato, può avere contribuito a determinare questa avanzata in riferimento al Trentino-Alto Adige. Regione in cui il fronte del SI è cresciuto non solo in valore assoluto ma anche in percentuale raccolta.

Colori scuri, seppur nelle già più sbiaditi, prevalgono poi in Lombardia, Piemonte e Veneto. In Liguria e Friuli-Venezia Giulia, invece, si osservano quasi ovunque gradazioni del grigio chiaro, che indicano arretramenti per il SI rispetto ai partiti pro-riforma alle europee. Un quadro abbastanza frastagliato emerge nelle quattro regioni dell'ex Zona Rossa: si notano sia comuni scuri in cui il SI ha raccolto (pochi) voti in più di PD, NDC-UDC e SE alle europee sia comuni chiari in cui invece è accaduto l'opposto. Con questi ultimi in netto sovrannumero.

Il vero tracollo per il fronte del SI si registra però al Sud. L'eccezione è il Molise, dove il fronte del SI cresce significativamente rispetto ai voti raccolti dai partiti di riferimento, tanto da fare segnare avanzate nei risultati percentuali e non solo sui valori assoluti. In buona parte della Sardegna e della Puglia il fronte del SI si dimostra capace di crescere, ma non più dell'affluenza, ed è quindi in calo come risultati percentuali. Lo stesso avviene nel Lazio nel vistoso caso romano. Ma nel resto del Sud il SI raccoglie meno voti dei partiti che hanno approvato la riforma nonostante l'aumento della partecipazione. 
FIG. 4 - Confronto per comune fra $i$ voti per il SI al referendum costituzionale e $i$ voti nelle europee 2014 ai partiti che hanno approvato la riforma costituzionale in Parlamento (PD, NCD-UDC, SE).

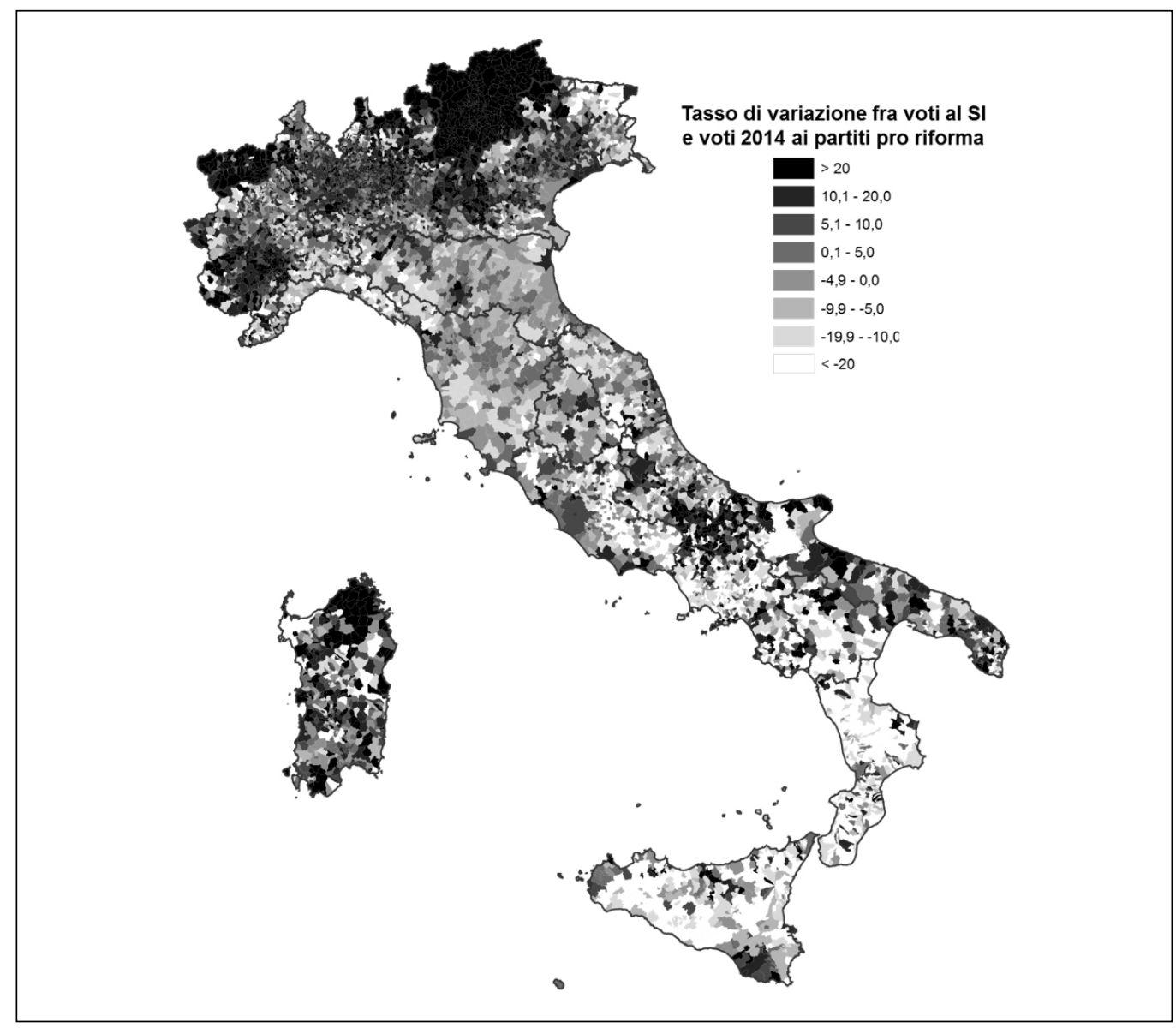

\section{Iflussi}

Ci concentriamo ora sull'analisi dei flussi elettorali in alcune città medio-grandi della penisola. Si tratta complessivamente di nove centri urbani così distribuiti: cinque al Nord (Torino, Genova, Milano, Brescia e Treviso), due nell'ex Zona Rossa (Parma e Ancona) e altri due al Sud (Napoli e Reggio Calabria). Come evidente, siamo in presenza di un campione che non può in alcun modo essere considerato rappresentativo dell' intero paese e dal quale non possiamo ricavare, per estrapolazione, generalizzazioni in chiave nazionale. Tuttavia le nostre nove città, alcune delle quali molto grandi, complessivamente contano oltre 4,5 milioni di abitanti pari al 7,5\% della popolazione italiana e possono lo stesso offrire un'indicazione importante, per quanto parziale, sui cambiamenti degli orientamenti di voto di una parte degli elettori.

In queste nove città il SI ha prevalso solo a Milano (51 a 49) e a Parma di un soffio $(50,3$ a 49,7). In tutte le altre il fronte del NO ha vinto, in alcuni casi stravinto, come a 
Napoli, dove ha ottenuto oltre il $68 \%$ dei voti, o a Reggio Calabria (quasi il $70 \%$ ), ma anche a Genova, dove il NO ha preso il 59\% dei voti, significativamente più che a Treviso $(55 \%)$. La partita è stata ben più aperta invece a Torino e Ancona dove il NO si è fermato a poco più del 53\% dei voti e soprattutto a Brescia, finita 48,4 a 51,6 per chi osteggiava la riforma.

I flussi elettorali ci permettono, attraverso una procedura di stima statistica dei coefficienti a partire dai dati delle singole sezioni elettorali di ciascun comune ${ }^{9}$, di inferire con un certo margine di approssimazione il comportamento di voto degli elettori dei vari partiti tra due elezioni nel tempo, o tra una elezione e una votazione referendaria come nel nostro caso. Nel caso in questione, mentre le variabili indipendenti resteranno i voti ai partiti rilevati in occasione di una precedente elezione, le variabili dipendenti saranno i voti raccolti dai due schieramenti: quello favorevole e quello contrario alla proposta di modifica costituzionale, più il bacino degli astenuti. Abbiamo scelto come punto di partenza le ultime elezioni per il rinnovo della Camera dei deputati svoltesi nel febbraio 2013, sostanzialmente in virtù del fatto che l'affluenza al referendum non si è fermata molto distante da quella di allora e in considerazione, inoltre, di quanto abbiamo sostenuto nei precedenti paragrafi sulla natura politica di questo referendum.

Osserviamo la Tabella 2 e vediamo come leggerla. In ciascuna riga per ogni singolo comune si trovano le destinazioni di voto degli elettori dei partiti nel 2013 (intestazioni di colonna). Il totale di ogni colonna di ciascuna città somma a 100 ed indica, fatti $100 \mathrm{i}$ voti ad un determinato partito nel 2013, in che misura questi si sono distribuiti a favore del SI, del NO o si sono astenuti. Per fare un esempio, a Torino, la colonna che riunisce gli elettori delle liste di Rivoluzione Civile e SEL del 2013, si è divisa quasi a metà, con una leggera prevalenza di contrari alla riforma ( 53 contro 47). La Tabella 3 va invece letta per riga. Fatto 100 i totali di riga, ci dice da dove provengono i voti ai due schieramenti referendari e al bacino dell'astensione. Uno sguardo complessivo alle due tabelle ci permette pur nella specificità delle singole realtà locali, di cogliere alcuni elementi comuni che con pochissime eccezioni emergono da tutti i casi qui presi in considerazione.

Iniziando la nostra discussione dal lato delle destinazioni (Tabella 2), possiamo notare come il comportamento di voto più compatto e omogeneo fra i diversi casi considerati sia quello degli elettori che nel 2013 avevano votato uno dei tre partiti della coalizione guidata da Mario Monti (SC, UDC, FLI). Questi hanno infatti votato SI, diversamente dallo stesso ex Presidente, in misura pressoché unanime. Con la sola eccezione di Reggio Calabria, in tutte le città almeno il 97\% degli elettori di Monti ha optato per la conferma delle modifiche alla Costituzione proposte.

Un altro minimo comun denominatore dei flussi nelle diverse città qui analizzate è la compattezza degli elettori del Movimento 5 Stelle sul fronte del NO. Eccezion fatta

9 Il metodo utilizzato per la stima dei coefficienti è il cosiddetto modello di Goodman (Goodman, 1953).

Per le singole città il valore redistribuito (VR) è risultato essere ben al di sotto del valore massimo convenzionalmente accettato. Nel dettaglio (Torino 3,9; Genova 2,8; Milano 3,7; Brescia 2,6; Treviso 6,1; Parma 3,5; Ancona 3,7; Napoli 4,5; Reggio Calabria 5,2). 
per il comune di Parma e in parte per Ancona, almeno 1'80\% degli elettori del partito di Grillo nel 2013 ha votato NO. I rimanenti si sono perlopiù astenuti dal voto e solo a Parma troviamo una percentuale importante, superiore al $10 \%$, che ha scelto le ragioni dello schieramento opposto.

Per quanto riguarda il PD, in tutto il Centro-nord ad esclusione quindi dei soli due comuni del meridione, circa due terzi degli elettori del 2013 ha votato per confermare la riforma: si va dal $60 \%$ di Genova al $71 \%$ di Milano e Brescia. A Napoli e Reggio Calabria grossomodo solo la metà degli elettori del $\mathrm{PD}$ ha seguito le indicazioni della maggioranza del partito. Questi dati ci mostrano quindi che una parte consistente, intorno a un terzo degli elettori che nel 2013 avevano scelto il PD guidato allora da Bersani, ha votato per affossare la riforma voluta dal suo successore alla segreteria.

Venendo al PDL, la maggioranza degli elettori ha scelto il NO, con la sola eccezione di Brescia. Escludendo la Leonessa d'Italia, la quota di elettori PDL che ha votato per respingere la riforma è compresa fra il 40 e il $60 \%$, mentre a Treviso raggiunge 1' $80 \%$. Di contro, tra un quinto e un terzo degli elettori 2013 del PDL ha avallato la proposta di modifica della costituzione. A Napoli sono stati solo il $18 \%$ e addirittura il $6 \%$ a Treviso. Una quota tutto sommato contenuta se consideriamo che Berlusconi e il suo partito, FI, avevano partecipato attivamente alla stesura del testo e votato a favore della riforma nelle commissioni delle due Camere e in aula fino alla terza lettura e che del PDL faceva parte anche NCD che la riforma l'ha votata fino in fondo e sostenuta al referendum. E in ogni caso troppo piccola perché i sostenitori del SI potessero sperare in un ribaltamento dell'esito referendario.

Maggiore variabilità nelle scelte di voto tra i comuni esaminati emerge se guardiamo alle dinamiche di flusso degli elettori di SEL e Rivoluzione Civile, che abbiamo aggregato per ragioni di opportunità statistica. Si noti come a Torino quasi la metà di questi elettori ha votato sì, un terzo a Milano così come a Reggio Calabria e tra il 25 e il 20\% nelle altre città, ma solo 1'11\% a Parma e praticamente nessuno a Brescia.

Guardando infine alla Lega Nord, occorre innanzitutto ricordate come alle politiche del 2013, guidata dall'allora segretario Roberto Maroni, abbia raccolto un consenso piuttosto limitato fermandosi a poco più del $4 \%$ su scala nazionale. Con la nuova segreteria, Salvini, ha impresso al partito una diversa linea marcandone maggiormente il profilo di protestatario e anti-politico, cavalcando alcuni temi in particolare, come l'euroscetticismo e la paura/rifiuto dell'immigrazione e crescendo fino al $6,2 \%$ delle europee del 2014. Oggi è accreditata dai vari istituti demoscopici mediamente attorno al $12 \%$. Questo per dire che la base elettorale della Lega Nord è molto cambiata dal 2013 e che gli elettori di allora, molto probabilmente sono diversi da quelli di oggi. Anche così si spiega il motivo per cui secondo i flussi elettorali stimati, gli elettori del Carroccio a Parma e a Torino si siano divisi in quote simili tra SI e NO con un leggero vantaggio dei secondi: $53 \%$ a Torino, $54 \%$ a Parma. Nelle altre città i NO hanno prevalso in modo molto netto: $84 \%$ a Milano, $85 \%$ a Treviso e $89 \%$ a Brescia. 
TAB. 2 - Flussi elettorali fra Politiche 2013 e referendum costituzionale 2016 in alcuni comuni, destinazioni.

\begin{tabular}{|c|c|c|c|c|c|c|c|c|c|c|}
\hline & \multirow[b]{2}{*}{$\begin{array}{c}\text { Voto } \\
\text { referendum } \\
\text { costituzionale }\end{array}$} & \multicolumn{9}{|c|}{ Voto Politiche 2013} \\
\hline & & $\begin{array}{c}\text { RC-SEL } \\
\%\end{array}$ & $\begin{array}{c}\mathrm{PD} \\
\%\end{array}$ & $\begin{array}{c}\text { Monti } \\
\%\end{array}$ & $\begin{array}{c}\text { PDL } \\
\%\end{array}$ & $\begin{array}{l}\text { Lega } \\
\%\end{array}$ & $\begin{array}{c}\text { Altri CD } \\
\%\end{array}$ & $\begin{array}{c}\text { M5S } \\
\%\end{array}$ & $\begin{array}{c}\text { Altri } \\
\%\end{array}$ & $\begin{array}{c}\text { Non voto } \\
\%\end{array}$ \\
\hline \multirow{3}{*}{ Torino } & SI & 47 & 64 & 100 & 27 & 47 & 100 & 0 & 25 & 0 \\
\hline & NO & 53 & 35 & 0 & 49 & 53 & 0 & 83 & 53 & 12 \\
\hline & Astensione & 0 & 2 & 0 & 24 & 0 & 0 & 17 & 22 & 88 \\
\hline \multirow{3}{*}{ Genova } & SI & 20 & 60 & 97 & 37 & & 62 & 0 & 57 & 0 \\
\hline & NO & 65 & 31 & 3 & 56 & & 38 & 89 & 18 & 7 \\
\hline & Astensione & 15 & 9 & 0 & 7 & & 0 & 11 & 25 & 93 \\
\hline \multirow{3}{*}{ Milano } & SI & 33 & 71 & 97 & 28 & 16 & 27 & 0 & 99 & 0 \\
\hline & NO & 55 & 27 & 0 & 58 & 84 & 63 & 80 & 0 & 9 \\
\hline & Astensione & 13 & 2 & 3 & 14 & 0 & 10 & 20 & 1 & 91 \\
\hline \multirow{3}{*}{ Brescia } & SI & 0 & 71 & 97 & 38 & 6 & 4 & 0 & 85 & 7 \\
\hline & NO & 61 & 28 & 3 & 35 & 89 & 96 & 86 & 15 & 12 \\
\hline & Astensione & 39 & 2 & 0 & 27 & 5 & 0 & 14 & 0 & 81 \\
\hline \multirow{3}{*}{ Treviso } & SI & 25 & 69 & 99 & 6 & 0 & 68 & 10 & 74 & 0 \\
\hline & NO & 29 & 31 & 1 & 82 & 85 & 0 & 90 & 18 & 3 \\
\hline & Astensione & 46 & 0 & 0 & 12 & 15 & 32 & 0 & 8 & 97 \\
\hline \multirow{3}{*}{ Parma } & SI & 11 & 69 & 100 & 20 & 46 & 49 & 17 & 85 & 0 \\
\hline & NO & 64 & 25 & 0 & 62 & 54 & 20 & 67 & 0 & 19 \\
\hline & Astensione & 25 & 6 & 0 & 18 & 0 & 31 & 16 & 15 & 81 \\
\hline \multirow{3}{*}{ Ancona } & SI & 21 & 65 & 100 & 39 & & 13 & 12 & 30 & 0 \\
\hline & NO & 58 & 22 & 0 & 61 & & 87 & 76 & 31 & 11 \\
\hline & Astensione & 22 & 13 & 0 & 1 & & 0 & 12 & 39 & 89 \\
\hline \multirow{3}{*}{ Napoli } & SI & 23 & 51 & 100 & 18 & & 24 & 0 & 0 & 0 \\
\hline & NO & 77 & 39 & 0 & 45 & & 36 & 100 & 38 & 11 \\
\hline & Astensione & 0 & 10 & 0 & 37 & & 41 & 0 & 62 & 89 \\
\hline \multirow{3}{*}{$\begin{array}{l}\text { Reggio } \\
\text { Calabria }\end{array}$} & SI & 33 & 56 & 54 & 31 & & 27 & 1 & 41 & 0 \\
\hline & NO & 67 & 18 & 46 & 52 & & 37 & 99 & 37 & 17 \\
\hline & Astensione & 0 & 26 & 0 & 17 & & 37 & 0 & 22 & 83 \\
\hline
\end{tabular}


Passiamo ora alle provenienze (Tabella 3). Innanzitutto, è importante notare la difficoltà dello schieramento del SI ad andare oltre la base di consenso del governo. In tutte le città tra il 71 e il $76 \%$ dei voti favorevoli alla proposta di modifica proviene da coloro che nel 2013 avevano votato il PD di Bersani o una delle liste che appoggiavano la candidatura dell'ex Presidente Monti (Scelta Civica, UDC, Futuro e Libertà per l'Italia). Per consistenza, il secondo flusso di voti in entrata per il SI, dopo quello legato ai partiti di governo, proviene dagli elettori del PDL che costituiscono, in misura variabile tra le varie città e con le vistose eccezioni di Parma, e soprattutto Treviso, tra il 10 e il 15\% dei voti del fronte del SI. Solo a Brescia si registra un minimo ingresso dal bacino del non voto (comunque inferiore a quello fatto segnare dallo schieramento avverso), mentre in tutte le altre città analizzate nessun astenuto del 2013 ha votato per confermare la riforma.

Se guardiamo, invece, al fronte del NO, ci accorgiamo di come nei diversi comuni considerati, con l'eccezione di quelli lombardi, fra il 40 e il $50 \%$ dei voti provenga dal bacino del M5S 2013. A Brescia e Milano questa quota scende al 30\% circa. Notiamo poi come, con la sola eccezione di Reggio Calabria, un sesto circa dei voti al NO provenga dal PD 2013: si tratta di una quota sostanzialmente analoga, anche se lievemente inferiore di quella proveniente dal PDL. Anzi, il contributo del PD al fronte del NO è superiore a quello del PDL a Torino, Genova e Brescia. Insomma le nostre analisi mostrano come, almeno nei grandi comuni qui analizzati, il ruolo della minoranza interna al PD sembri essere stato rilevante per le sorti complessive del voto referendario.

In conclusione, l'analisi dei dati di flusso qui condotta ci dice che si è trattato principalmente di un voto "politico", dove gli schieramenti sui due fronti referendari hanno in buona sostanza rispecchiato quelli a sostegno dell'azione del governo Renzi, mostrando, i sostenitori della riforma, scarsa capacità di raccogliere consensi al di fuori di quest'area e gli avversari, invece, una notevole compattezza. 
TAB. 3 - Flussi elettorali fra Politiche 2013 e referendum costituzionale 2016 in alcuni comuni, provenienze.

\begin{tabular}{|c|c|c|c|c|c|c|c|c|c|c|c|}
\hline & \multirow{3}{*}{$\begin{array}{c}\text { Voto } \\
\text { referendum } \\
\text { costituzionale }\end{array}$} & \multicolumn{9}{|c|}{ Voto Politiche 2013} & \multirow[b]{2}{*}{ Totale } \\
\hline & & RC-SEL & $\mathrm{PD}$ & Monti & PDL & Lega & $\begin{array}{l}\text { Altri } \\
\mathrm{CD}\end{array}$ & M5S & Altri & $\begin{array}{l}\text { Non } \\
\text { voto }\end{array}$ & \\
\hline & & $\%$ & $\%$ & $\%$ & $\%$ & $\%$ & $\%$ & $\%$ & $\%$ & $\%$ & $\%$ \\
\hline \multirow{3}{*}{ Torino } & SI & 8 & 43 & 29 & 11 & 3 & 6 & 0 & 2 & 0 & 100 \\
\hline & NO & 8 & 20 & 0 & 17 & 3 & 0 & 42 & 3 & 8 & 100 \\
\hline & Astensione & 0 & 1 & 0 & 10 & 0 & 0 & 2 & 11 & 75 & 100 \\
\hline \multirow{3}{*}{ Genova } & SI & 3 & 48 & 26 & 15 & & 5 & 0 & 3 & 0 & 100 \\
\hline & NO & 7 & 17 & 1 & 15 & & 2 & 52 & 1 & 5 & 100 \\
\hline & Astensione & 2 & 7 & 0 & 3 & & 0 & 8 & 1 & 79 & 100 \\
\hline \multirow{3}{*}{ Milano } & SI & 4 & 43 & 30 & 12 & 2 & 1 & 0 & 8 & 0 & 100 \\
\hline & NO & 7 & 17 & 0 & 26 & 12 & 3 & 29 & 0 & 6 & 100 \\
\hline & Astensione & 2 & 2 & 1 & 8 & 0 & 1 & 9 & 0 & 78 & 100 \\
\hline \multirow{3}{*}{ Brescia } & SI & 0 & 47 & 27 & 14 & 2 & 0 & 0 & 6 & 4 & 100 \\
\hline & NO & 6 & 17 & 1 & 12 & 21 & 5 & 29 & 1 & 7 & 100 \\
\hline & Astensione & 6 & 2 & 0 & 15 & 2 & 0 & 0 & 7 & 69 & 100 \\
\hline \multirow{3}{*}{ Treviso } & SI & 2 & 42 & 34 & 2 & 0 & 3 & 5 & 11 & 0 & 100 \\
\hline & NO & 2 & 15 & 0 & 24 & 14 & 0 & 41 & 2 & 1 & 100 \\
\hline & Astensione & 6 & 0 & 0 & 5 & 4 & 2 & 0 & 2 & 81 & 100 \\
\hline \multirow{3}{*}{ Parma } & SI & 1 & 48 & 23 & 6 & 3 & 2 & 10 & 6 & 0 & 100 \\
\hline & NO & 7 & 18 & 0 & 19 & 3 & 1 & 40 & 0 & 12 & 100 \\
\hline & Astensione & 4 & 5 & 0 & 7 & 0 & 2 & 13 & 1 & 67 & 100 \\
\hline \multirow{3}{*}{ Ancona } & SI & 3 & 47 & 26 & 13 & & 1 & 8 & 2 & 0 & 100 \\
\hline & NO & 8 & 14 & 0 & 17 & & 7 & 46 & 2 & 6 & 100 \\
\hline & Astensione & 4 & 11 & 0 & 0 & & 0 & 10 & 3 & 71 & 100 \\
\hline \multirow{3}{*}{ Napoli } & SI & 7 & 44 & 31 & 15 & & 3 & 0 & 0 & 0 & 100 \\
\hline & NO & 10 & 16 & 0 & 18 & & 2 & 39 & 2 & 12 & 100 \\
\hline & Astensione & 0 & 3 & 0 & 12 & & 2 & 3 & 0 & 80 & 100 \\
\hline \multirow{3}{*}{$\begin{array}{l}\text { Reggio } \\
\text { Calabria }\end{array}$} & SI & 8 & 40 & 15 & 24 & & 6 & 1 & 7 & 0 & 100 \\
\hline & NO & 7 & 6 & 6 & 18 & & 3 & 41 & 3 & 17 & 100 \\
\hline & Astensione & 0 & 8 & 0 & 6 & & 3 & 0 & 2 & 81 & 100 \\
\hline
\end{tabular}




\section{Conclusioni}

I dati presentati in questo artico mostrano come il voto referendario sia stato un vero e proprio voto "politico", e che questa politicizzazione abbia segnato le sorti della riforma costituzionale. L'alta partecipazione elettorale, la geografia del voto, anche le differenze emerse nel voto fra grandi centri e piccoli comuni che abbiamo osservato nella Figura 3, sono tutte caratteristiche che richiamano elezioni politiche.

Altre se ne possono aggiungere. Ad esempio, abbiamo detto del caso di Milano, dove il Si ha prevalso sul NO. Ma se guardiamo più in profondità, ci accorgiamo di come il SI abbia vinto largamente nei quartieri centrali e più agiati ma ha perso in quelli periferici. Lo stesso fenomeno si riscontra anche a Roma e a Torino, dove complessivamente il NO ha prevalso, tranne che nei quartieri del centro. $\mathrm{O}$ ancora possiamo citare come nei 100 comuni con la percentuale più alta di disoccupati abbia vinto il NO con quasi i due terzi dei voti. Nei 100 comuni con la più bassa disoccupazione, al contrario, è stato il SI a sfiorare il 60\%. Insomma sembra emergere un legame fra voto al NO e marginalità sociale.

Anche l'analisi dei flussi ci conferma questa politicizzazione del voto. Gli elettori dei partiti di governo hanno votato per il SI. Tutti gli altri hanno massicciamente respinto la riforma. Anzi, si segnalano defezioni più forti verso il NO fra i tradizionalmente molto fedeli elettori del PD, che non verso il SI tra quelli dei partiti per il NO. Addirittura, nelle grandi città qui analizzate, la quota di elettori del NO proveniente dal PD non è molto inferiore a quella che nel 2013 aveva votato PDL.

Una volta che il referendum aveva assunto caratteri politici, e associata la riforma a Renzi e al suo governo, il destino della riforma era spacciato. Innanzitutto alla luce della congiuntura internazionale, in cui gli elettori delle democrazie occidentali appaiono assai poco propensi a votare soluzioni proposte dal mainstream politico - si vedano i successi del Brexit e di Trump. A questa si somma poi la specifica congiuntura italiana, quella di un governo non particolarmente popolare che proponeva al paese la riforma. In tanti elettori è inevitabilmente scattato un riflesso partigiano. La notevole omogeneità del risultato della partecipazione al voto denota che questo voto è stato percepito dagli elettori come se si trattasse di una elezione politica vera e propria, anche se non erano in lizza né partiti né candidati. Una prova ulteriore viene dal buon risultato del SI nelle regioni dell'ex Zona Rossa, il tradizionale bacino di consensi delle forze della sinistra italiana. Dove il $\mathrm{PD}$ è più forte, il SI è andato meglio. Ma il problema è che il PD rimane forte solo in una zona limitata. Così, il bilancio complessivo recita che il SI ha prevalso in 12 province su 106, di cui 11 fra Emilia-Romagna e Toscana. Se, come ci mostrano le analisi dei flussi elettorali, a votare per il SI sono gli elettori del PD e dei suoi alleati di governo (con i primi poi neanche così compatti), mentre gli elettori di tutti gli altri partiti votano per il NO, il destino del voto referendario è inevitabilmente segnato a sfavore della riforma. Riforma che invece godeva di un certo apprezzamento trasversale per i suoi contenuti, cosa che però non è contato al momento di votare visto che altri fattori, quelli legati alla politica nazionale, e alla sopravvivenza del governo Renzi in particolare, erano in gioco.

Infatti, i contenuti della riforma, durante la sua gestazione parlamentare, godevano di un consenso abbastanza ampio presso il corpo elettorale. Così era ancora al momento 
della sua approvazione. Confidando forse su questo favore trasversale, Renzi ha personalizzato il voto referendario nel tentativo di conseguirne una ulteriore legittimazione, dopo quella ormai distante 30 mesi delle europee, in vista del finale di legislatura. Oppure lo ha fatto nella convinzione che la propria figura avesse una popolarità in grado di aiutare la vittoria del SI: se così è stato, i risultati elettorali gli hanno chiaramente dato torto.

In ogni caso, la conseguenza è stata che in un voto politicizzato l'importanza relativa dei contenuti della riforma nel determinare le scelte di voto si è fortemente ridotto, mentre una quota sempre crescente di elettori si convinceva a votare in virtù del proprio favore (o sfavore) nei confronti del governo. Era difficile evitare questa associazione, ma il premier è stato incauto nel rendere la cosa più facile ai suoi avversari ed a facilitarne la mobilitazione. L'associazione ci sarebbe comunque stata, almeno in parte, così come suggerito dalla letteratura sulle elezioni di secondo ordine, in virtù delle scelte strategiche di chi alla riforma si opponeva. Tuttavia, l'impostazione della campagna del SI ha certamente favorito il successo delle strategie dei rivali, e incrementato la quota di elettori che hanno scelto sulla base del governo più che della riforma in sé. Inoltre, ha favorito la mobilitazione degli avversari di Renzi interni al partito. Infatti, un ulteriore elemento che merita di essere sottolineato è la spaccatura all'interno del bacino 2013 del PD che emerge dalle nostre analisi sui flussi elettorali. Anche in questo caso, una scelta strategica meno personalizzante e divisiva ma che cercasse invece di ottenere il sostegno attivo della minoranza interna avrebbe potuto contribuire al successo della riforma.

Così, nei mesi intercorsi dall'approvazione al voto referendario, il favore verso i contenuti della riforma registrato dai sondaggi non si trasformava poi in voti. Naturalmente, è assolutamente possibile che $\mathrm{i}$ sondaggi fossero in errore, e gli elettori italiani non fossero affatto favorevoli ai contenuti della riforma. Ma in realtà vi sono anche rilevazioni immediatamente precedenti il voto che testimoniano la contemporanea intenzione di votare NO, e il gradimento per i contenuti della riforma presso gli stessi intervistati: una chiara dimostrazione della scissione fra opinione circa la riforma e intenzione di voto al referendum. Ecco come, per via della scarsa popolarità del governo al momento in cui si è svolta la consultazione referendaria, governo che però aveva messo la propria testa in palio nel voto referendario, e che non è stato capace di fare sostenere il SI neppure da tutti coloro i quali la riforma l'avevano approvata, si viene determinando il pesante rapporto di forza di 3 voti per il NO ogni due voti per il SI registrato nel territorio italiano.

In conclusione, possiamo rilevare come il risultato del referendum si inquadri perfettamente in una elezione di secondo ordine nel momento centrale del ciclo politico nazionale. Le prospettive per le opzioni sostenute dai partiti di governo sono particolarmente plumbee. In effetti, l'alta partecipazione elettorale registrata potrebbe fare pensare che questo referendum non sia stata una elezione di secondo ordine. Ma, in realtà, la posta in gioco era particolarmente alta, se paragonata con altre elezioni di secondo ordine, per cui è in linea con le attese della teoria che il calo della partecipazione rispetto alle politiche sia più contenuto che, ad esempio, in occasione di consultazioni locali o europee. Ciò detto, il risultato mostra chiaramente la scarsa salute elettorale delle forze di governo. Se a questa sommiamo la sostanziale spaccatura all'interno dell'elettorato del PD, che per circa un terzo ha votato $\mathrm{NO}$, ecco che il risultato osservato il 4 dicembre appare perfettamente spiegato. 


\section{Riferimenti bibliografici}

Bellucci, P. 2006. «All'origine della popolarità del governo in Italia, 1994-2006». Rivista Italiana di Scienza Politica 36 (3): 479-504.

Campbell, A. 1960. «Surge and Decline: A Study of Electoral Change». Public Opinion Quarterly 24 (3): 397-418.

Cataldi, M., V. Emanuele e N. Maggini. 2015. «2014. Un anno di elezioni regionali: il filotto del PD». Quaderni dell'Osservatorio elettorale 73: 159-175.

Crainz, G. e C. Fusaro. 2016. Aggiornare la Costituzione. Storia e ragioni di una riforma. Roma, Donzelli.

D’Alimonte R. 2016. «Referendum, avanti il No. Ma sul merito italiani favorevoli». Il Sole 24 Ore, 17 novembre 2016.

De Sio, L., V. Emanuele e N. Maggini (a cura di). 2014. Le elezioni europee 2014. Dossier CISE (6), Rome, CISE.

Diamanti, I. 2016. «Referendum, il No avanza. Il Sì è indietro di 7 punti». La Repubblica, 17 novembre 2016.

Emanuele, V., N. Maggini e A. Paparo (a cura di). 2016. Cosa succede in città? Le elezioni comunali 2016. Dossier CISE (8), Rome, CISE.

Goodman, L. A. 1953. «Ecological regression and behavior of individual». American Sociological Review 18: 663-664.

Marsh, M. 1998. «Testing the Second-Order Election Model after Four European Elections». British Journal of Political Science 28 (4): 591-607.

Miller, W. L., e Mackie, M. 1973. «The Electoral Cycle and the Asymmetry of Government and Opposition Popularity: An Alternative Model of the Relationship Between Economic Conditions and Political Popularity». Political Studies 21 (3): 263-279.

Moran, P. A. P. 1950. «Notes on Continuous Stochastic Phenomena». Biometrika 17(1): 17-23.

Pagnoncelli, N. 2016. «Referendum, il No avanti al 52\%. Al Sud la maggioranza dei contrari». Il Corriere della Sera, 2 ottobre 2016.

Paparo, A. e M. Cataldi (a cura di). 2015. Dopo la luna di miele: Le elezioni comunali e regionali fra autunno 2014 e primavera 2015. Dossier CISE (7), Rome, CISE.

Reif, K., e H. Schmitt. 1980. «Nine Second-Order National Elections-a Conceptual Framework for the Analysis of European Election Results». European Journal of Political Research 8 (1): 3-44.

Schmitt, H. 2005. «The European Parliament Elections of June 2004: Still Second-Order?». West European Politics 28 (3): 650-679.

Schmitt, H. e E. Teperoglou. 2015. «The 2014 European Parliament Elections in Southern Europe: Second-Order or Critical Elections?». Southern European Society and Politics 20 (3): 287-309.

Shugart, M. S. 1995. «The Electoral Cycle and Institutional Sources of Divided Presidential Government». American Political Science Review 89 (02): 327-343. 
Stimson, J. A. 1976. «Public Support for American Presidents A Cyclical Model». Public Opinion Quarterly 40 (1): 1-21.

Tufte, E. R. 1975. «Determinants of the Outcomes of Midterm Congressional Elections». American Political Science Review 69 (03): 812-826.

Zagrebelsky, G. e F. Pallante. 2016. Loro diranno, noi diciamo. Vademedum sulle riforme istituzionali. Bari, Laterza. 